\title{
Geometric Discretisation of the Toda System
}

\author{
Adam Doliwa \\ Istituto Nazionale di Fisica Nucleare, Sezione di Roma \\ P-le Aldo Moro 2, I-00185 Roma, Italia \\ and \\ Institute of Theoretical Physics, Warsaw University \\ ul. Hoża 69, 00-681 Warsaw, Poland *
}

\begin{abstract}
The Laplace sequence of the discrete conjugate nets is constructed. The invariants of the nets satisfy, in full analogy to the continuous case, the system of difference equations equivalent to the discrete version of the generalized Toda equation.

Keywords: Integrable systems, discrete geometry, Toda equation
\end{abstract}

Preprint ROME1-1160/96, October 27, 1996

Dipartimento di Fisica, Università di Roma "La Sapienza"

I.N.F.N. - Sezione di Roma

*Permanent address 


\section{Introduction}

Among all the integrable lattice systems a particular role is played by the discrete analogue of the generalized Toda system [1][2] [3]. Its suitable limits with respect to the lattice parameters give rise to the various types of soliton equations including the Kadomtsev-Petviashvili equation, Kortewegde Vries equation, Benjamin-Ono equation, sine-Gordon equation or their differential-difference versions. Moreover, the discrete Toda system provides unification of many particular examples of solvable models of statistical physics and quantum field theory [4] [6].

To construct integrable discretisation of the given soliton equation there have been used several different approaches, for example:

- discrete version of the Lax system [7]

- Hirota's method via the bilinear form [2]

- extension of the Zakharov - Shabat dressing method [1]

- direct linearisation using linear integral equations [8].

Recently another discretisation approach has been applied to those of soliton equations which describe geometrically meaningful objects such as curves or some special types of surfaces [9]-[12]. As a working principle, the discrete analogues of the relevant geometric properties must be found.

This approach has been used to discretise Toda system. In section 2, we collect useful facts from the theory of the conjugates nets (CNs) 13 and recall their connection with the Toda system [14]. In section 3, we construct the Laplace sequence of the discrete conjugate nets (DCNs). In the section 4 , we define invariants of the sequence and write down a difference equation

relating the invariants of the neighboring DCNs which is the discrete version of the Toda system.

\section{Conjugate nets, their Laplace transform and Toda system}

Definition 1. Two directions tangent to the surface $M \subset \mathbb{E}^{3}$ in point 
$\mathbf{x} \in M$ are called conjugate when they are conjugate with respect to the Dupin indicatrix for the point [13].

Fact 1. Given a curve $\gamma$ on a surface $M \subset \mathbb{E}^{3}$. The ruled surface made out of lines conjugate to tangent directions to $\gamma$ is developable, i.e. it is formed by the tangent directions to a curve $\gamma_{1}$.

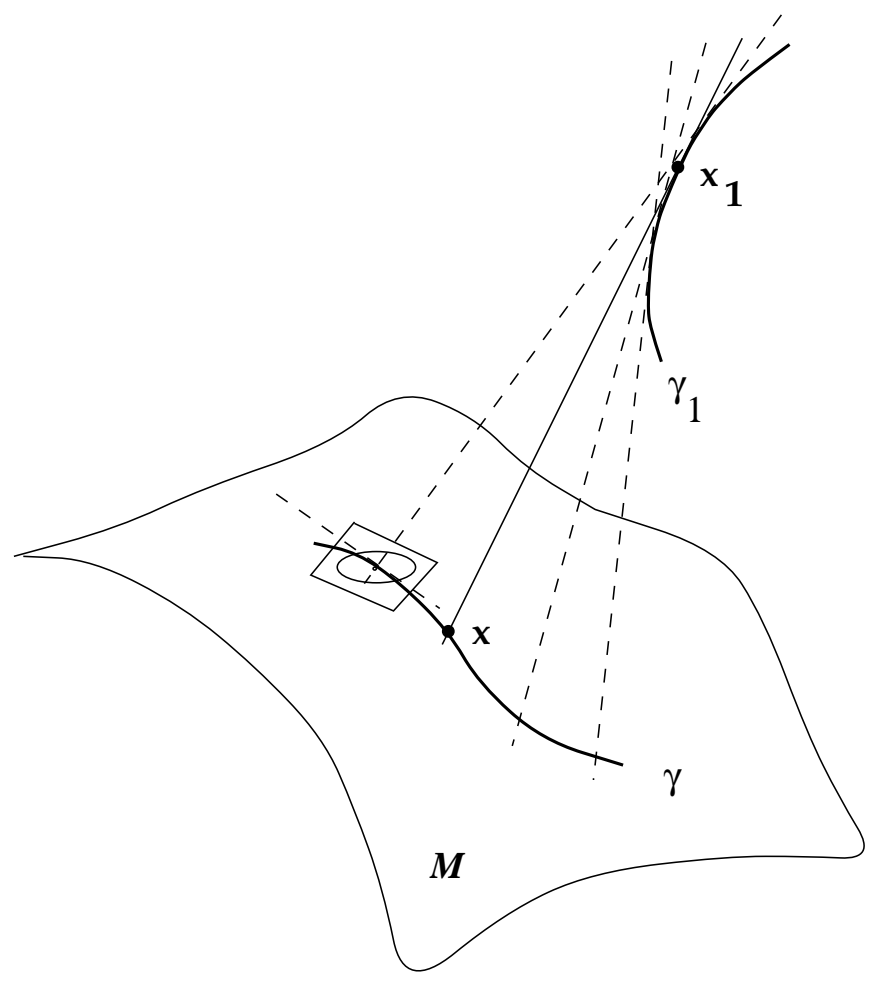

Fig. 1

Definition 2. Two families of curves on a surface $M \subset \mathbb{E}^{3}$ are said to form conjugate net if the tangents to a curve of each family at their point of intersection have conjugate directions.

The curves of the first family due to the Fact 1 . give rise to a surface $M_{1}$. Similarly, the curves of the second family give rise to a surface $M_{-1}$.

Fact 2 The points of both new surfaces which correspond to initial families of curves on $M$ form again conjugate nets. 
Similarly, $M_{1}$ gives rise to a new conjugate net $M_{2}$ (one can show that $\left.M_{(1)-1}=M\right)$. This way we obtain (in general infinite) sequence of conjugate nets

$$
\ldots, M_{-2}, M_{-1}, M_{0}=M, M_{1}, M_{2}, \ldots
$$

called the Laplace sequence. The analytic expressions for these results are based on the following

Fact 3. A necessary and sufficient condition for the parametric curves of coordinates $(u, v)$ to form a conjugate system is that the second mixed derivative of the vector $\mathbf{x}(u, v)$ pointing the surface is tangent to the surface.

This means that the vector-function $\mathbf{x}(u, v)$ satisfies the Laplace equation

$$
\mathbf{x}_{, u v}+a \mathbf{x}_{, u}+b \mathbf{x}_{, v}=0,
$$

where $a$ and $b$ are determinate functions of the parameters $u, v$, and comma denotes differentiation.

To construct the surface $M_{1}$ we use the lines of constant $v$ on $M$. Then the points of $M_{1}$ are given by

$$
\mathbf{x}_{\mathbf{1}}=\mathbf{x}+\lambda \mathbf{x}_{, u},
$$

where $\lambda$ is a determinate function of the parameters $u, v$. But the direction from $\mathbf{x}$ to $\mathbf{x}_{1}$ must be tangent to the line $u=$ const. on $M_{1}$

$$
\mathbf{x}_{1, v}=\mu \mathbf{x}_{, u},
$$

where again $\mu$ is a function of $u, v$.

As a consequence of the equations (11), (2) and (3) we obtain an overdetermined system

$$
\left(\lambda_{, v}+a \lambda-\mu\right) \mathbf{x}_{, u}+(1-b \lambda) \mathbf{x}_{, v}=0,
$$

which implies

$$
\lambda=\frac{1}{b} \quad, \quad \mu=\left(\frac{1}{b}\right)_{, v}-\frac{a}{b} .
$$

Finally

$$
\begin{aligned}
\mathbf{x}_{\mathbf{1}} & =\mathbf{x}+\frac{1}{b} \mathbf{x}_{, u}, \\
\mathbf{x}_{\mathbf{1}, v} & =\left(\left(\frac{1}{b}\right)_{, v}-\frac{a}{b}\right) \mathbf{x}_{, u},
\end{aligned}
$$


and similarly

$$
\begin{aligned}
\mathbf{x}_{-\mathbf{1}} & =\mathbf{x}+\frac{1}{a} \mathbf{x}_{, v} \\
\mathbf{x}_{-\mathbf{1}, u} & =\left(\left(\frac{1}{a}\right)_{, u}-\frac{b}{a}\right) \mathbf{x}_{, v} .
\end{aligned}
$$

To proceed further we are forced to leave the domain of the theory of surfaces in the Euclidean space $\mathbb{E}^{3}$. It turns out that the notion of the conjugate net is a projective property, i.e. when the whole space is subjected to the projective transformation $\mathrm{CNs}$ are transformed into CNs. As the Cartesian coordinates of the points of the surface and functions $a, b$ change, it is important to know the projective invariants of the conjugate nets. Such invariant functions are

$$
\begin{aligned}
& k=b_{, v}+a b \\
& h=a_{, u}+a b .
\end{aligned}
$$

Let us find the relation between the invariants of the Laplace sequence of conjugate nets. It is easy to show, using equations (6), that the vectorfunction $\mathbf{x}_{\mathbf{1}}$ satisfies the Laplace equation again (proving the Fact 2)

$$
\mathbf{x}_{\mathbf{1}, u v}+\frac{k}{b} \mathbf{x}_{\mathbf{1}, u}+\left(b-\left(\ln \frac{k}{b}\right)_{, u}\right) \mathbf{x}_{\mathbf{1}, v}=0,
$$

which implies the form of the transformed coefficients $a, b$

$$
\begin{aligned}
a_{1} & =\frac{k}{b} \\
b_{1} & =b-\left(\ln \frac{k}{b}\right)_{, u} .
\end{aligned}
$$

In consequence, the Laplace transform of the invariants reads

$$
\begin{aligned}
& h_{1}=k, \\
& k_{1}=2 k-(\ln k)_{, u v}-h .
\end{aligned}
$$

Finally, the sequence $k_{l}$ of invariants satisfies the system of equations

$$
(\ln k)_{, u v}=-k_{l+1}+2 k_{l}-k_{l-1} \quad, \quad l=\ldots-2,-1,0,1,2, \ldots
$$


known in this context to Darboux [15], and equivalent to the standard form of the Toda system

$$
\theta_{l, u v}=-\mathrm{e}^{\theta_{l+1}-\theta_{l}}+\mathrm{e}^{\theta_{l}-\theta_{l-1}},
$$

where

$$
k_{l}=\mathrm{e}^{\theta_{l+1}-\theta_{l}} .
$$

The next two sections are devoted to the discretisation of all the reasoning presented above.

\section{Discrete conjugate nets and their Laplace sequences}

Definition 3. By a discrete conjugate net we mean a map $\mathbf{X}: \mathbb{Z}^{2} \rightarrow \mathbb{E}^{3}$ such that all the elementary quadrilaterals

$$
\langle\mathbf{X}(i, j), \mathbf{X}(i+1, j), \mathbf{X}(i, j+1), \mathbf{X}(i+1, j+1)\rangle
$$

are planar (see [16]).

The above definition is a natural discretisation of the basic property of CNs described in the Fact 3. The discrete analogue of the Laplace equation (1) is

$$
\begin{aligned}
\mathbf{X}(i+1, j+1) & =\mathbf{X}(i, j)+A(i, j)(\mathbf{X}(i+1, j)-\mathbf{X}(i, j)) \\
& +B(i, j)(\mathbf{X}(i, j+1)-\mathbf{X}(i, j)) .
\end{aligned}
$$

From now on, for any function $F$ on $\mathbb{Z}^{2}$ we will use the following convention

$$
F_{p}^{q}=F(i+p, j+q)
$$

for example, the above equation (16) can be rewritten as

$$
\mathbf{X}_{1}^{1}=\mathbf{X}+A\left(\mathbf{X}_{1}-\mathbf{X}\right)+B\left(\mathbf{X}^{1}-\mathbf{X}\right)
$$

The intersection points of the directions of the opposite edges of the elementary quadrilateral (they may intersect at the plane in infinity — we must take into consideration the projective picture) define points of the Laplace trans-

forms of the net, denoted by $\tilde{\mathbf{X}}$ and $\check{\mathbf{X}}$ (see Fig. 2 where also the convention of labelling points of the new nets is given). 


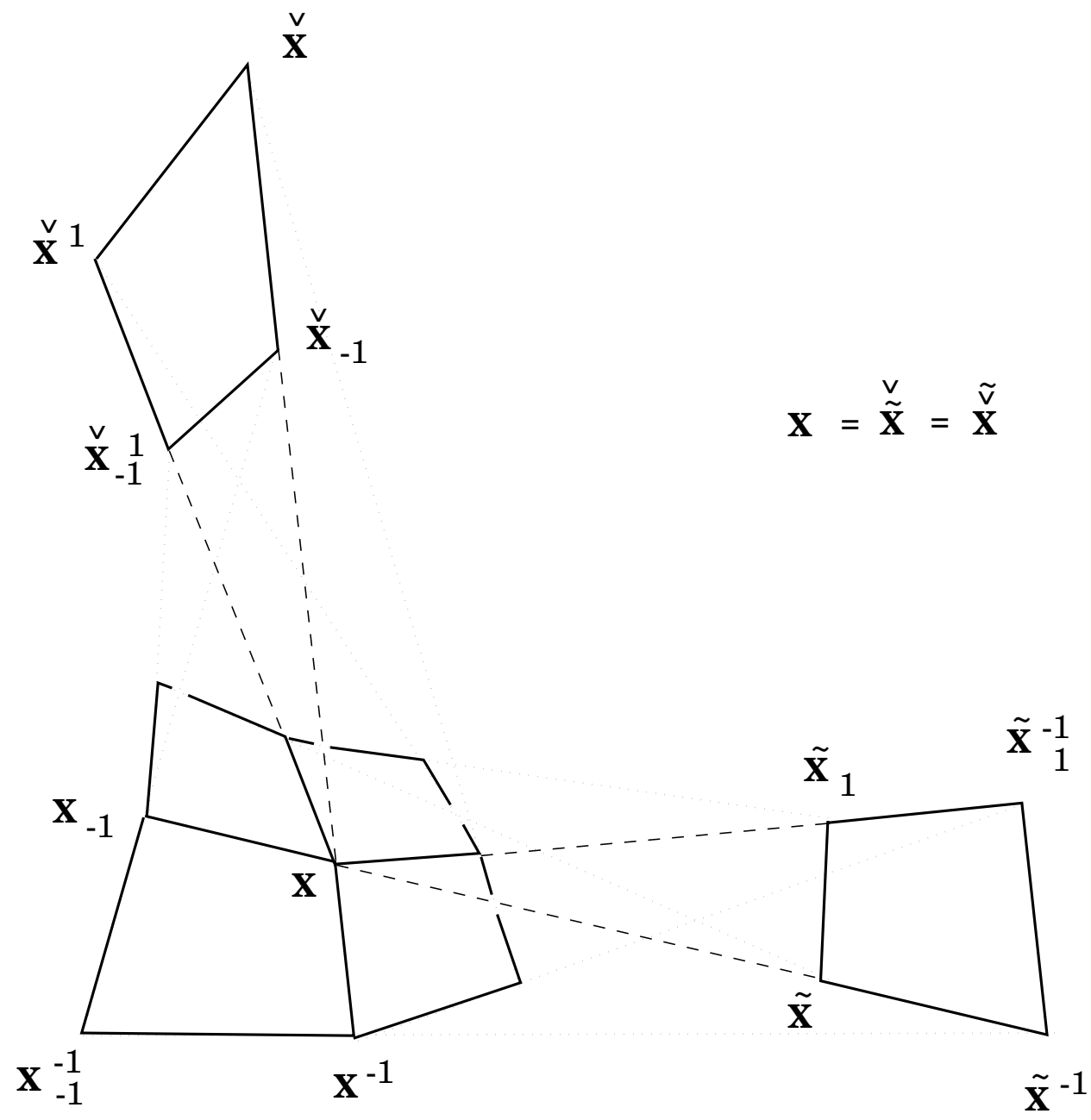

Fig. 2

The following two facts can be easily examinated.

Fact 4. Both Laplace transforms $\tilde{\mathbf{X}}$ and $\check{\mathbf{X}}$ of the discrete conjugate net $\mathbf{X}$ are again discrete conjugate nets.

Fact 5. The Laplace transformations of the discrete conjugate nets do not branch, i.e. $\tilde{\tilde{X}}=\tilde{\check{X}}=\mathbf{X}$. 
This way any DCN gives rise to a whole sequence (in general infinite on both sides) of DCNs.

In what follows, we define the analogues of the functions $\lambda, \mu$ and find the Laplace transforms of the coefficients $A, B$.

$$
\begin{aligned}
\tilde{\mathbf{X}} & =\mathbf{X}_{-1}+\Lambda\left(\mathbf{X}-\mathbf{X}_{-1}\right) \\
\tilde{\mathbf{X}}-\tilde{\mathbf{X}}^{-1} & =M\left(\mathbf{X}-\mathbf{X}_{-1}\right)
\end{aligned}
$$

Similarly as for CNs we can express functions $\Lambda, M$ in terms of $A$ and $B$

$$
\begin{aligned}
\Lambda_{1} & =\frac{A}{1-B}, \\
M_{1}^{1} & =\frac{A^{1}}{1-B^{1}}-\frac{1}{1-B} .
\end{aligned}
$$

Let us discuss, for a moment, the limit of the small lattice parameter $\varepsilon$ (in general one can take two independent lattice parameters)

$$
\begin{aligned}
\mathbf{X}_{1}-\mathbf{X} & \simeq \varepsilon \mathbf{X}_{, u} \\
\mathbf{X}^{1}-\mathbf{X} & \simeq \varepsilon \mathbf{X}_{, v} \\
\mathbf{X}_{1}^{1}-\mathbf{X}^{1}-\left(\mathbf{X}_{1}-\mathbf{X}\right) & \simeq \varepsilon^{2} \mathbf{X}_{, u v}
\end{aligned}
$$

In consequence

$$
\begin{aligned}
1-A & \simeq \varepsilon a \\
1-B & \simeq \varepsilon b \\
\Lambda & \simeq \frac{\lambda}{\varepsilon}, \\
M & \simeq \mu .
\end{aligned}
$$

The equations (18) can be used to obtain the discrete Laplace equation for

the net $\tilde{\mathbf{X}}$ (proving the Fact 4 ) and allow to find the transformed coefficients $\tilde{A}$ and $\tilde{B}$

$$
\begin{aligned}
\tilde{A} & =\frac{\Lambda_{1}^{1}}{\Lambda_{1}^{1}-M_{1}^{1}}, \\
\tilde{B} & =\frac{M_{1}^{1}}{M^{1}} \cdot \frac{M^{1}-\Lambda^{1}+1}{M_{1}^{1}-\Lambda_{1}^{1}} .
\end{aligned}
$$

Similar considerations can be made also for the second Laplace transform $\check{\mathbf{X}}$ of the conjugate net $\mathbf{X}$. 


\section{Invariants of the discrete conjugate nets and the discrete Toda system}

The fundamental property of the projective transformations is that straight lines are transformed into straight lines and consequently planes into planes. This implies that the notion of discrete conjugate net is in fact projective notion, and Laplace sequences are mapped into Laplace sequences od DCNs.

In looking for the discrete analogues of the invariants $k$ and $h$ let us recall that the basic projective invariant is the cross-ratio of four points $A, B, C, D$ on the line

$$
Q(A, B, C, D)=\frac{\overrightarrow{A B}}{\overrightarrow{B C}} \div \frac{\overrightarrow{A D}}{\overrightarrow{C D}},
$$

where the ratio of two vectors should be understood as a proportionality factor.

\section{Remark 1.}

$$
Q(A, B, C, D)=Q(D, C, B, A)
$$

In our construction of the discrete conjugate nets and their Laplace sequences there are two natural systems of four points on the line (see Fig. 2), and we propose for the invariants

$$
\begin{aligned}
& K=Q\left(\mathbf{X}_{-1}, \mathbf{X}, \tilde{\mathbf{X}}, \tilde{\mathbf{X}}^{-1}\right) \\
& H=Q\left(\mathbf{X}^{-1}, \mathbf{X}, \check{\mathbf{X}}, \check{\mathbf{X}}_{-1}\right)
\end{aligned}
$$

Equations (18) and (19) allow to express the invariant $K$ in terms of the coefficients $A, B$ of the Laplace equation

$$
K=\frac{M}{(\Lambda-1)(M-\Lambda)}=\frac{A_{-1} B_{-1}^{-1}}{A_{-1}+B_{-1}-1}-1,
$$

and similarly

$$
H=\frac{B^{-1} A_{-1}^{-1}}{A^{-1}+B^{-1}-1}-1 .
$$

In the limit of the small lattice parameter we obtain

$$
\begin{aligned}
& K \simeq \varepsilon^{2} k \\
& H \simeq \varepsilon^{2} h .
\end{aligned}
$$


To construct the discrete Toda system we need to find the Laplace transforms of the invariants. Using formulas (19), (22), (26) and (27) we derive the following relations

$$
\begin{gathered}
\tilde{H}=K, \\
\tilde{K}_{1}+1=\frac{\left(K_{1}^{1}+1\right)(K+1)}{H^{1}+1} \frac{K_{1} K^{1}}{K_{1}^{1} K} .
\end{gathered}
$$

The first of the above equations is just the consequence of the Remark 1 . Finally, the equation (30) rewritten in terms of functions $K(i, j, l)$ (we go back to the full definitions)

$$
\ldots, K(i, j,-1)=\check{K}, K(i, j, 0)=K, K(i, j, 1)=\tilde{K}, \ldots
$$

gives rise to the difference system

$$
\frac{(K(i+1, j, l+1)+1)(K(i, j+1, l-1)+1)}{(K(i+1, j+1, l)+1)(K(i, j, l)+1)}=\frac{K(i+1, j, l) K(i, j+1, l)}{K(i+1, j+1, l) K(i, j, l)} .
$$

The above equations in the limit of the small parameter $\varepsilon$ reduce to the equations (13). They are equivalent to the known in the literature gauge invariant discrete version of the Toda system (see e.g. [5]).

Remark 2. In all the above calculations we had not used essentially the fact that $\mathbf{X}$ is a point of the space of dimension 3. All the results remain valid if we consider the ambient space of the bigger dimension.

Remark 3. The transformation of a given discrete Laplace equation to a new such equation can be considered also as a discrete analog of the cascade method (proposed by Laplace) of solving the equation. This method is especially useful when the Laplace sequence terminates on a surface degenerated to a curve.

Remark 4. The finite Laplace sequences correspond to the open Toda systems, which are related to the $A$-type simple Lie algebras, periodic Laplace sequences are related in turn to the affine $A^{(1)}$ Lie algebras. Various additional geometric structures in the ambient space allow to find the open and periodic Toda systems related to other simple Lie algebras (see e.g. [18, [19]). This procedure can be applied also to the discrete Toda system and should recover equations existing in the literature [ [1] [17]. 


\section{Acknowledgments}

I would like to thank A. Sym for pointing out reference 16 and to P. M. Santini for stimulating discussions. This work was supported partially by the Committee of Scientific Research (KBN) under the Grant Number 2P03 B 18509 .

\section{References}

[1] D. Levi, L. Pilloni and P. M. Santini, J. Phys. A 14 (1981), 1567-1575.

[2] R. Hirota, J. Phys. Soc. Jpn. 50 (1981), 3785-3791.

[3] E. Date, M. Jimbo and T. Miwa, J. Phys. Soc. Jpn. 51 (1982), 41254131.

[4] A. Kuniba, T. Nakanishi and J. Suzuki, Int. Journ. Mod. Phys. A 9 (1994) 5215-5312.

[5] I. Krichever, O. Lipan, P. Wiegmann and A. Zabrodin, Quantum Integrable Systems and Elliptic Solutions of Classical Discrete Nonlinear Equations, preprint ESI 330 (1996), hep-th/9604080.

[6] I. G. Korepanov, Algebraic integrable dynamical systems, 2+1 dimensional models in wholly discrete space-time, and the inhomogeneous models in 2-dimensional statistical physics, solv-int/9506003.

[7] M. J. Ablowitz and J. F. Ladik, J. Math. Phys. 16 (1975) 598.

M. J. Ablowitz and J. F. Ladik, J. Math. Phys. 17 (1976) 1011.

M. J. Ablowitz, Stud. Appl. Math. 58 (1978) 17.

[8] G. R. W. Quispel, F. W. Nijhoff, H. W. Capel and J. van der Linden, Physica 125 A, (1984) 344.

F. W. Nijhoff, H. W. Capel, G. L. Wiersma and G. R. W. Quispel, Phys. Lett. 103 A (1984) 293.

V. G. Papageorgiu, F. W. Nijhoff and H. W. Capel, Phys. Lett. 147 A (1990) 106.

[9] A. Doliwa and P. M. Santini, J. Math. Phys. 36 (1995), 1259-1273. 
[10] A. Doliwa and P. M. Santini, The integrable dynamics of discrete and continuous curves, Preprint IFT 22/94, Warsaw (to appear in Proceedings of Esterel Conference on Symmetries of Discrete Equations, May 1994).

[11] A. Bobenko and U. Pinkall, Discrete Surfaces with constant Negative Gaussian Curvature and the Hirota equation, Sfb 288 Preprint 127 (1994), to appear in J. Diff. Geom.

[12] A. Bobenko and U. Pinkall, J. reine angew. Math. 475 (1996), 187-208.

[13] L. P. Eisenhart, A Treatise on the Differential Geometry of Curves and surfaces, Ginn and Company, Boston.

[14] J. Weiss, Phys. Lett. A 137 (1989), 365-368.

[15] G. Darboux, Leçons sur la théorie générale des surfaces II, GauthierVillars, Paris, 1888.

[16] R. Sauer, Differenzengeometrie, Springer, Berlin, 1970.

[17] R. S. Ward, Phys. Lett. A 199 (1995) 45-48.

[18] A. Doliwa, Holomorphic Curves and Toda Systems, preprint IFT UW 7/95, to appear in Lett. Math. Phys.

[19] A. Doliwa, Harmonic Maps and Periodic Toda Systems, preprint IFT UW 10/95, to appear in J. Math. Phys. 